\title{
Nanomedicine for COVID-19: Potential of Copper Nanoparticles
}

\author{
Keelan Jagaran ${ }^{1 \mathbb{D}}$, Moganavelli Singh ${ }^{1 *}$ (i) \\ 1 Nano-Gene and Drug Delivery Laboratory, Discipline of Biochemistry, University of KwaZulu-Natal, Private Bag \\ X54001, Durban, South Africa \\ * Correspondence: singhm1@ukzn.ac.za;
}

Scopus Author ID 3709591900

Received: 15.10.2020; Revised: 10.11.2020; Accepted: 11.11.2020; Published: 14.11.2020

\begin{abstract}
The COVID-19 pandemic has fuelled a global demand to establish novel diagnostic and treatment options, apart from the search for a vaccine. This, coupled with the need to mitigate the spread of infections, called for large-scale strategies, including alternative anti-viral methods, together with classical disinfection and prevention protocols. One such approach exists in the form of the essential micronutrient, copper. Copper surfaces have been observed to exhibit viral survival of fewer than 4 hours, compared to cardboard, stainless steel, and plastic with an average survival time of 24 hours, 48 hours, and 72 hours, respectively. Copper's natural ability to aid in the functioning of critical immune cells, including B cells, natural killer cells, T helper cells, macrophages, and neutrophils, make it a potential therapeutic agent against SARS-CoV-2, both internally and externally to the host. This antiviral property can be enhanced by the generation of copper nanoparticles for use in nanomedicine. Copper nanoparticles can generate reactive oxygen species that interfere with viral operations and disrupt the viral membrane. This review paper focuses on copper nanoparticles for favorable therapeutic outcomes, together with its targeted interaction with cells expressing the angiotensin-converting enzyme 2 (ACE2), for the treatment of COVID-19.
\end{abstract}

Keywords: COVID-19; SARS-CoV-2, copper; nanoparticles; nanomedicine; therapy.

(C) 2020 by the authors. This article is an open-access article distributed under the terms and conditions of the Creative Commons Attribution (CC BY) license (https://creativecommons.org/licenses/by/4.0/).

\section{Introduction}

The outbreak of Coronavirus Disease 2019 (COVID-19) caused by the severe acute respiratory syndrome coronavirus $2(\mathrm{SARS}-\mathrm{CoV}-2)$ has emerged as the latest threat impacting all citizens' global health. This life-threatening disease has placed a critical strain on research, public health, medical communities, resources and has become an economic burden worldwide. The transition from a Public Health Emergency of International Concern, declared on the $30^{\text {th }}$ January 2020, to a pandemic on $11^{\text {th }}$ March 2020, resulted from a 13-fold increase in infected individuals and an increasing number of affected countries. With millions of infected individuals and a rising death rate, there is an urgency for further studies and treatment options to mitigate this disease.

This novel strain of coronavirus $(\mathrm{CoV})$, preceded by the severe acute respiratory syndrome (SARS)-CoV, and the Middle East respiratory syndrome (MERS)-CoV, primarily targets the respiratory system of infected individuals. Consequently, COVID-19 is associated with mild to severe pneumonia, progressing to Acute Respiratory Distress Syndrome (ARDS), which is the leading cause of mortality [1,2]. Molecular testing has been carried out with the WHO recommending specimen collections from the lower respiratory tract, including the 
endotracheal aspirate, expectorated sputum or bronchoalveolar, and the upper respiratory tract. Diagnosis is also obtained via serological testing, laboratory examinations, and imaging [1]. Current treatment options rely on anti-virals and vaccines to curb the spread of the virus [2]. To date, no specific medicines or vaccines have been developed to prevent or treat COVID-19, with self-care through aggressive isolation measures being implemented, predominately, worldwide: novel and different approaches aimed at addressing COVID-19 needs to be thoroughly researched and established.

One such approach is the exploitation of copper for its anti-viral properties, which was brought to light and widely utilized since the nineteenth century as a disinfectant [3]. The SARS-CoV-2 remains viable for up to 4 hours on solid copper compared to plastic and stainless steel. It has viability times from 2 to 3 days and cardboard for up to 24 hours [4]. In the body, copper is known to play a vital role in the immune system's maintenance and function via its ability to produce specific antibodies, aid in cell-mediated immunity, and kill infectious microbes. This is achieved through its involvement in the functions of neutrophils, macrophages, T helper cells, natural killer cells, and B cells [5]. With recent advancements in medicine, specifically in nanomedicine, novel treatment options may become available. Nanomedicine can be an attractive alternative to traditional medicine, exhibiting efficiency in its delivery, with the added advantage of low cost. Nanomedicine had sparked interest in clinical practice due to the use of nanoparticles (NPs) as delivery vehicles for gene and drug therapy, in addition to their diagnostic and therapeutic potential. Nanoparticles allow for the efficient transfer of genes and drugs with maximum efficacy, increased bioavailability, and target specific cells/tissue with controlled-release pharmacokinetics [6,7].

Nanomedicine has recently advanced to formulate a novel treatment option, utilizing cellular membranes, presenting targeted proteins. This approach is contradictory to many others because it does not target the causative agents, thus focusing on the affected host cells instead. Upon membrane binding to the NP, the NP possesses the ability to express proteins that the SARS-CoV-2 virus requires for its infectivity [7]. These NPs or better described as nanosponges, absorb these viruses, thereby minimizing cellular infectivity and subsequently discouraging the CoV contagion as well [7].

Hence, nanomaterials can be modified to be utilized in pharmacology for diagnostic purposes, together with the prevention, control, and targeted treatment of diseases [8]. With copper portraying anti-viral properties in its natural state, it can be hypothesized that exploiting this property at a nanoscale may result in more significant therapeutic outcomes. This review is thus aimed at exploring nanomedicine with an emphasis on CuNPs as a viable approach in the fight against COVID-19.

\section{SARS-CoV-2}

The SARS-CoV-2 virus took the world by storm, wreaking havoc in almost every area of the economic and health sectors. This single-stranded positive-sense RNA virus belongs to the family of Coronaviridae [9]. These viruses are naturally found as either pleomorphic, enveloped, or spherical particles, each ranging between $150-160 \mathrm{~nm}$ in size [10]. The virus (Figure 1) presents with a genome size of $\sim 30$ kilobases encoding essential viral proteins for successful entry into host cells, as a result of the spike glycoprotein (S), as well as to counteract the hosts immune responses via a viral suppressor protein of RNAi (VSR), expressed by the nucleocapsid protein (N) [10]. Epidemiologically, the SARS-CoV-2 shares a $93.1 \%$ identity with the bat coronavirus RaTG13 for the spike gene. 


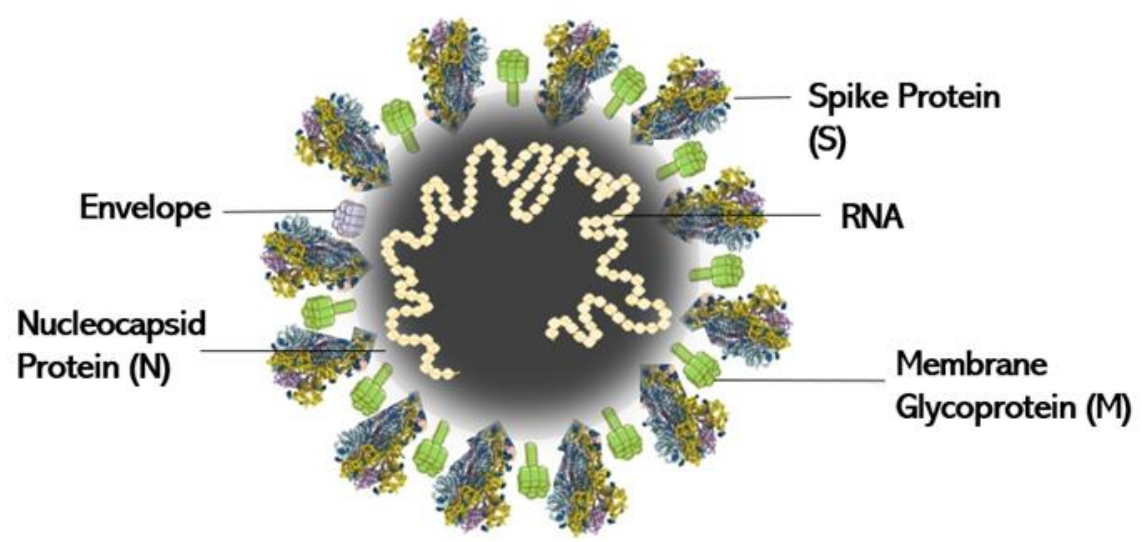

Figure 1. Schematic diagram of SARS-CoV-2.

\subsection{Mechanism of infection.}

Following subsequent preliminary studies based on full-length genomic phylogenetic analysis, the similarities between the SARS-CoV and SARS-CoV-2 viruses were identified, thereby aiding research into understanding their mechanisms of infection. The mechanism elucidated for SARS-CoV implicates subunits S1 and S2 of the homotrimeric spike glycoprotein to play a primary role in cellular invasion, starting from binding the glycoprotein to the cellular membrane and triggering a cascade of events. Binding of the virus is attained primarily via the Angiotensin-converting enzyme 2 (ACE2), a mechanism that has been previously elucidated. This results in the dissociation of S1 from ACE2, followed by the conversion of S2 from a metastable pre-fusion to a post-fusion state $[11,12]$.

Computer modeling of the SARS-CoV-2 virus portrayed the receptor-binding motif $(\mathrm{RBM})$ role in the interaction between the spike trimer and ACE2 expressed on the host cells. The SARS-CoV-2 RBD is composed of a five-stranded, twisted, antiparallel $\beta$ sheet $(\beta 1, \beta 2$, $\beta 3, \beta 4$, and $\beta 7$ ), with the core structure being formed by short connecting helices as well as loops [13]. It had been elucidated that an extended insertion is found between the $\beta 4$ and $\beta 7$ strands present in the core, which contains the short $\beta 5$ and $\beta 6$ strands, together with loops and the $\alpha 4$ and $\alpha 5$ helices. This includes the residues involved in the interaction between the ACE2 protein and the SARS-CoV-2 [9]. The ACE2 proteins contain an $\mathrm{N}$-terminal peptidase domain, each with two lobes, with the lower lobe involved in the interaction [14]. The successful interaction is visualized as a concave exterior surface of the RBM, which exhibits the ability to accommodate the N-terminal helix of the ACE2.

\subsection{Clinical manifestations.}

Clinical manifestations related to SARS-CoV-2 are non-specific to infected individuals, with some individuals being asymptomatic. In contrast, others present with mild to severe pneumonia, resulting in death [15]. As the virus progresses through the ACE2 proteins in the respiratory tract and moves towards the lungs, a significant increase in inflammation is noted, together with fluid intake into the air sacs or alveoli. Alternatively, cytokine storms play a crucial role in pneumonia manifestation, indicating a flared out immune response, causing apoptosis of healthy cells in the lungs, and allowing fluid intake [16]. Complications of COVID-19 infections include acute heart injury as well as Acute Respiratory Distress Syndrome (ARDS) [15]. 
In a recent study, the symptoms of COVID-19 were elucidated and ranked from the most to the least common manifestation. These symptoms included fever (98\%), cough (76\%), fatigue (44\%), and myalgia (44\%). Furthermore, atypical symptoms caused includes sputum (28\%), headache (8\%), hemoptysis (5\%) and diarrhoea (3\%) [17].

\subsection{Host defense and treatment.}

It is crucial that the host defense mechanisms against SARS-CoV-2 must be completely elucidated for an efficacious vaccine to be created. It has been understood that the host elicits both cell-mediated immune responses as well as humoral responses. The latter response was shown to produce effective antibodies against the $\mathrm{S}$ and $\mathrm{N}$ proteins of the SARS-CoV [18]. Although this response was seen to have a beneficial effect in fighting off COVID-19, the antibodies produced are short-lived and are thus deemed ineffective in some cases [19].

As a result of the natural defense mechanism being unable to eradicate the SARS-CoV2 , treatment options in the form of drugs and genes are thus imperative. Presently, there are no specific anti-virals and vaccines available to treat COVID-19, with treatment being administered to the symptoms presented. The initial step involves oxygen therapy; however, in complicated cases, non-invasive (NIV) and invasive mechanical ventilation (IMV) are recommended to treat respiratory failure, refractory to the initial oxygen therapy.

\section{Copper}

Copper $(\mathrm{Cu})$ is an important trace element, which is absorbed in the small intestine into a plasma pool. This element plays a critical role in the function and maintenance of the immune system, specifically for B cells' functioning, natural killer cells, T helper cells, macrophages, and neutrophils [5]. Hence, $\mathrm{Cu}$ is essential in cell-mediated immunity and the production of specific antibodies in response to the infectious microbes.

Following a study conducted by the US National Institute of Health (NIH), and as mentioned earlier, viral survival was shorter on $\mathrm{Cu}$ surfaces compared to cardboard, stainless steel, and plastic [4]. The effect of $\mathrm{Cu}$ is seen to be time and concentration-dependent, resulting in irreversible damage to the virus and destruction of the cell walls [20]. This action is achieved via three mechanisms: (1) disruption of the viral membrane and envelop, thus destroying the RNA of the virus, (2) providing interference to the essential proteins required for the virus to operate, and (3) generation of reactive oxygen species (ROS) which inevitably kills the virus, as demonstrated by the SARS-CoV [21-23].

Furthermore, studies conducted based on inflammatory markers and $\mathrm{Cu}$ levels suggest that $\mathrm{Cu}$ plays a role in the fight against inflammation. This was seen by an increase in serum and liver $\mathrm{Cu}$ concentrations among adjuvant-induced arthritis in rats [24]. Although there is a lack of knowledge concerning the exact mechanism involved, it was observed that an increase in interleukin 6 (IL6) resulted in increased production and secretion of ceruloplasmin, a Cucarrying protein [25]. This is specifically supportive in COVID-19 cases due to the high serum ferritin (iron stores) levels exhibited in patients. The elucidation of the role of ceruloplasmin has been portrayed as an iron trafficker, thereby balancing the enterocytes' ferritin levels [26]. $\mathrm{Cu}$ can induce autophagy in response to a viral infection. This mechanism provides cells with the ability to survive external stress caused by the virus. In the case of $\mathrm{Cu}$, it has been elucidated that induction of this mechanism and formation of autophagic vacuoles, together with 
apoptosis, is as a result of the presence of $\mathrm{Cu}$ in the hosts' body. These discoveries have portrayed $\mathrm{Cu}$ as an ideal candidate and a potential therapeutic agent [26].

For therapeutic use, the toxicity of $\mathrm{Cu}$ to humans was described as being very rare as a result of homeostatic mechanisms that reduce excessive $\mathrm{Cu}$ intake. $\mathrm{Cu}$ supplementation, however, presents with adverse effects when taken in excessive quantities (>10 mg/day) in a short period [27]. For optimum $\mathrm{Cu}$ absorption, a diet of decreased fiber, ascorbic acid, divalent metals, zinc, iron, fructose, and cysteine with increased protein intake and polybasic amino acids has been advised [28]. Cu thus portrays favorable results against viruses, in general, with a potential therapeutic role against SARS-CoV-2 specifically. This dietary intake and supplementation of $\mathrm{Cu}$ in the body provide greater immunity towards COVID-19. $\mathrm{Cu}$ in this form, however, cannot be conjugated to other therapeutics, such drugs or genes, for enhanced viral elimination, necessitating novel approaches to the use of $\mathrm{Cu}$. One such approach is in exploiting the area of nanomedicine.

Before the entry of the virus to the host, surface contamination poses a significant threat in the transmission of COVID-19. This was identified following an increased number of positive cases amongst healthcare personals, despite using proper personal protective equipment (PPE) and following strict barrier precautions [29]. $\mathrm{Cu}$ has been identified as a material that inactivates the virus within a shorter period, compared to other commonly used material such as stainless steel and plastic [4]. This was further confirmed by a prior study investigating the CoV-229E strain. This study observed that brass containing a minimum of $70 \% \mathrm{Cu}$ was more effective against $\mathrm{CoV}-229 \mathrm{E}$ and that the efficacy increased upon using higher percentages of $\mathrm{Cu}[20]$. This inactivation was attributed to the ROS generated by the $\mathrm{Cu}$ ions [4]

\section{Nanomedicine}

Nanomedicine, an amalgamation of medicine and nanotechnology, utilizes NPs in diagnostic or therapeutic functions [30]. Diagnostically, these NPs, being the most coveted systems, possess the ability to visualize pathologies, track disease progressions from the onset, providing a means for early detection. Alternatively, NPs can also serve as therapeutic agents, acting as successful transporters, carrying pharmacologically active agents, and releasing them in a controlled manner at desired diseased sites. These NPs exhibit low cytotoxicity in vivo, together with the relative ease of administration [31]. These beneficial properties should be leveraged in the fight against COVID-19. This can be achieved via; (i) the creation of highly sensitive and specific diagnostic tools, (ii) a highly efficient gene and drug delivery vehicle, (iii) ultra-fine filters for face masks, (iv) advancements in tools for contact tracing, and (v) novel coating of surfaces to minimize viral survival time. NPs have been seen to inhibit the viral infectivity, irreversibly, through the permanent damage caused to the virion of the virus [32]. It is due to this that NPs exhibit the significant therapeutic potential to curb the spread and mortalities caused by SARS-CoV-2. The idea of incorporating potent biological agents, together with metal nanoparticle carriers, is rapidly gaining momentum for the treatment of various diseases [33]. These potent drugs can be used to interfere with the interaction of the virus and the ACE2 receptor, inhibiting the entry of the virus. Furthermore, to exploit nanomedicine, researchers are looking at an area of applied nanodiagnosis for managing COVID-19 [34]. 


\subsection{Copper nanoparticles.}

With the SARS-CoV-2 infections escalating at an alarming rate, and an influx in hospital admissions, research-based on treatment strategies is imperative. As previously mentioned, $\mathrm{Cu}$ elicits robust anti-viral properties, allowing it to be a suitable candidate in the treatment of SARS-CoV-2 infections. Furthermore, nanomedicine is emerging as a strong candidate in its ability to fight off infections, in general. Hence, studies on copper nanoparticles (CuNPs) can provide insight into a novel therapeutic strategy that could be highly beneficial in the challenges facing COVID-19 treatment.

CuNPs in medicine has been studied to a lesser extent to its other metallic counterparts. However, they have been utilized in nanofluids, in the facilitation of increased heat transfer, together with their application in catalysis or photocatalysis $[35,36]$. Due to their potential anti-viral property, low toxicity, antifungal, and antibacterial activity, excellent biocompatibility, oxidation resistance, and higher availability at lower costs, CuNPs may be exploited as attractive alternatives in nanomedicine, together with other metallic NPs such as silver $(\mathrm{Ag})$ and gold $(\mathrm{Au})$ ) [37,38]. CuNPs also possess optical and magnetic properties, together with a high surface to volume ratio [39]. CuNPs contains a single phase tenorite, exhibiting a narrow bandgap of $1.7 \mathrm{eV} \mathrm{[40].} \mathrm{This} \mathrm{is} \mathrm{essential} \mathrm{to} \mathrm{its} \mathrm{ability} \mathrm{to} \mathrm{be} \mathrm{used} \mathrm{in} \mathrm{medical}$ applications, owing to the properties mentioned above [41]. Previous reports also confirmed the ability of CuNPs to be easily coated with polymers for greater stability for in vivo. This provides a gateway for the successful conjugation of pharmacologically active agents for gene and drug delivery.

The antimicrobial nature of $\mathrm{Cu}$ should be duly exploited for its use in the development of effective PPEs. Copper nanoparticles, in particular, could be utilized and incorporated in the equipment such as respirators, surgical gowns, non-woven shoes, and so on, to mitigate the risk of infection by healthcare personnel. This significantly reduces the viability of the virus through simple carrier interfaces, thereby controlling the spread of the disease [42]. Studies and trials on the use of CuNPs, or synergistic NPs encompassing varied metallic shell or core, is thus imperative.

The biomedical applicability of CuNPs has been seen in many sectors of medicine related to cancer, viral and bacterial studies. Following a survey conducted by the United States environmental protection agency (EPA), $\mathrm{Cu}$ and its alloys have been discovered as the first efficacious metallic antimicrobial agent [3]. Cu's role as an anti-viral agent has been studied in two independent studies $[43,44]$, both portraying $\mathrm{Cu}$ as a highly efficient and novel treatment against the hepatitis $\mathrm{C}$ virus (HCV) and Herpes simplex virus 1 (HSV-1). The former study based on HCV suggested an interference of entry and attachment of the infectious virion to the hepatic host cells as a result of an oxide of CuNPs, termed copper oxide nanoparticles (CuONPs). Furthermore, an $83.3 \%$ inhibition rate of the HSV-1 following the subsequent administration of CuONPs in vitro was reported [44]. This inhibition was possibly due to interference caused by the NPs in the viral replication stage.

CuNPs have portrayed significant results in the inhibition of viruses in a highly efficient manner, providing a platform for further, more intensive studies to be conducted, especially with the challenges posed by COVID-19. The current standing of the pandemic necessitates the search for a vaccine and treatment, with CuNPs serving as a potential candidate to be considered. 


\subsection{Nanosponges.}

A significant development in nanomedicine can be seen in the formulation of nanosponges. These nanomaterials are made up of nanoparticle cores, surrounded by cellular membranes, as seen in Figure 2. As a result of this conjugation, a three-dimensional network is formed, which has advantages such as gradual degradation and superior drug absorption/complexation properties [45]. Furthermore, the molecular architecture of the nanosponges allows for the co-encapsulation of one or more drugs for direct and effective treatment [46].

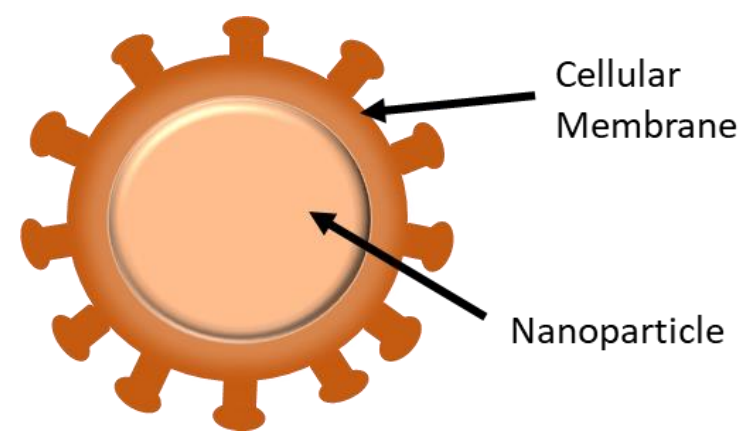

Figure 2. Representation of a nanosponge that is comprised of a nanoparticle coated with a cellular membrane, preferably of human lungs.

Pore-forming toxins (PFTs) have shown the ability to disrupt the cellular membranes of the infected host and increasing its permeability. This is a necessary action in the case of bacterial infections and constitutes an important virulence control mechanism [47]. This action paved the way for research into nanosponges, especially the use of red blood cell (RBC) membrane-coated nanoparticles. The nanoparticle core is vital to the success of the nanosponges to enable prolonged circulation times [48]. The nanosponges mimic RBCs and attract the attack by toxins. In this process, the toxins are removed by absorption by the nanosponges out of the bloodstream.

This approach has been recently modified to treat SARS-CoV-2, with direct treatment of affected host cells, rather than treating the causative agents. This novel treatment has been inspired by the ability of the SARS-CoV-2 to invade the host cell via its protein receptors. The development of nanosponges against COVID-19 is dependent on the nanoparticles being coated with human-cell-derived membranes, such as human lung epithelial type II cells and human macrophage cells [7]. These cells can be conjugated to the nanoparticle core while expressing the ACE 2 and CD147 proteins, which are crucial for viral entry into cells. The expression of these proteins was visualized using western blot analysis of the nanosponges, together with viral receptors present in the natural state of the cellular membranes. The study confirmed the facilitation of nanosponges in membrane protein retention as well as showing a lack of contamination from other intracellular proteins [7]. Hence, the ability of these nanosponges to neutralize the virus in a concentration-dependent manner can be considered a potential treatment.

The absorption of the SARS-CoV-2 may be coupled with the beneficial properties exhibited by $\mathrm{Cu}$ and $\mathrm{CuNPs}$. CuNPs can be utilized as the intended nanosponge core to provide greater efficacy in absorbing and possibly eradicating the virus as well. In instances when the SARS-CoV-2 virus mutates, the nanosponges provides a means of treating the infection, irrespective of mutational change. It is thus proposed that the use of $\mathrm{Cu}$ would significantly 
add to the beneficial properties demonstrated by the nanosponges, allowing for a secondary killing mechanism to be present in vivo. Hence, further research in this area and clinical trials are required with the hope of a successful and highly efficient treatment for all infected individuals.

\subsection{Drug therapy.}

With outstanding advancements being made in medicine regularly, gene and drug therapy has portrayed results which are highly advantageous in the treatment of various diseases. Before the discovery of nanomedicine, drugs have been and continue to be utilized. The major limitation in drug efficacy is due to low solubility, leading to lowered diffusion into targeted cells. This limits the bio-accessibility of the drug following oral intake and creates a requirement for a higher concentration of the drug by intravenous routes [49]. Drugencapsulated NPs were thus established to overcome this hurdle for a targeted therapeutic approach. NP based drug therapy can be regarded as an advanced technology in medicine. This approach provides a means to improve therapeutic indices by enhancing properties such as solubility, diffusivity, immunogenicity, bioavailability, targeted and controlled drug release in vivo. As a result of the successful modification, an extended cycle of drug survival has been noted, together with a substantial decline in side effects as a result of reduced toxicity, improved biodistribution, and general ease of administration [50].

Drug delivery can occur via passive or self-delivery, differing by the location of the drug to the NP. Passive delivery requires the drug to be incorporated into the inner cavity/core of the NPs. Upon reaching their specific targeted sites, these NPs begin to disintegrate, thereby releasing the drug cargo. Alternatively, the direct conjugation of the drug to the NPs allows for self-delivery to occur. This delivery relies on the drug being released at precise times for maximum efficacy [51]. The delivery of these drug-encapsulated NPs can be further classified according to how they attach and enter specific cells, viz. active and passive delivery. The former requires the coupling of antibodies and peptides to the NPs, for active receptor binding of the targeted site. The latter mechanism is based on the NP freely circulating the bloodstream; however, these are controlled via properties such as temperature, molecular sites, $\mathrm{pH}$, and shape. Due to the tumor microenvironment, drug delivery to tumors often utilize the enhanced permeability and retention (EPR) effect for passive delivery.

With regards to COVID-19, studies have portrayed the role of SARS-CoV-2 in producing a hyperinflammatory state, as characterized by a fulminant cytokine storm, known as hypercytokinemia. This phenomenon results in acute respiratory distress syndrome (ARDS), leading to death in more severe cases [2]. With low success rates being identified with the administration of inflammatory inhibitors, the use of a multidrug NP, encapsulating adenosine (Ad), and tocopherol (VitE) to reduce the cytokine storm was proposed [52]. The study concluded that this approach was a promising therapeutic, yielding more positive results compared to conventional antioxidant and Ad therapy. With the success of this approach, the proposition of utilizing CuNPs as a delivery vehicle remains true for greater efficacy, with a reduction in toxicity.

Drug therapy using CuNPs may also increase the efficacy of anti-viral drugs, which possess the potential to treat COVID-19, as illustrated in Figure 3. These anti-viral drugs include ritonavir/lopinavir, remdesivir, and arbidol, each portraying its mechanism of action 
by acting as protease inhibitors, causing premature termination of RNA and blocking of viral fusion, respectively [53-55].

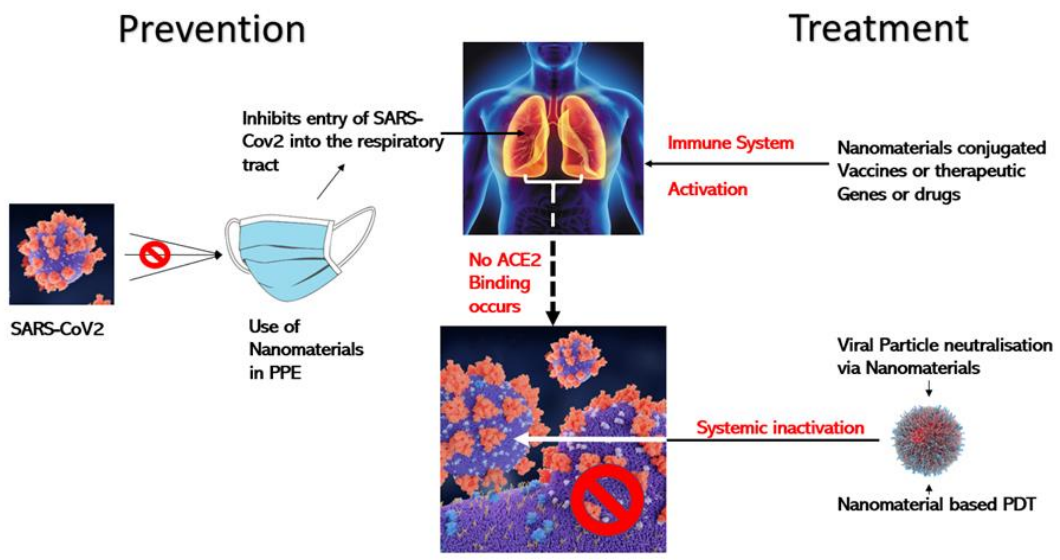

Figure 3. A representation of the role of nanomaterials in the potential prevention and treatment of COVID-19. Their use in preventative measures, such as employing nanoparticles in PPE, portrays its role in curbing the entry of the virus into the respiratory system. Therapeutically, nanoparticles can be delivered, with conjugated drugs or genes via the pulmonary system, or administration followed by the subsequent photodynamic therapy (PDT) to inactivate SARS-CoV-2 through inhibition of cellular binding via ACE2.

\subsection{Gene therapy.}

Understanding gene function, genetic aberrations, and biological processes have brought this therapy to light to create a treatment targeting specific genes. Gene therapy has been established in many clinical developments for a vaccine against SARS-CoV-2. This therapeutic approach is characterized into three classes of vaccines employing genes, that is, DNA, viral vectors, and mRNA [56]. Various studies have been undertaken to mitigate the harsh impact of COVID-19, each focusing mostly on the SARS-CoV-1 spike (S) protein. This is generally undertaken via the encoding of the open reading frame present in the $S$ gene of the gene fragment. The studies conducted have completed the initial first phases of testing, with phase II possessing the ability to provide evidence of efficacy and immunogenicity [56].

Gene therapy aims at precise modification, deletion, or replacement of so-called "faulty" genes in diseased cells [57]. One aspect of gene therapy employs recombinant nucleic acids possessing therapeutic genes, which results in the targeted mRNA being translationally blocked, thereby reducing disease progression [58] by a process known as gene silencing. These nucleic acids include small interfering RNA (siRNA), plasmid DNA (pDNA), DNAzymes, microRNA (miRNA), and antisense oligonucleotides (antisense ODNs) [59]. CRISPR (clusters of regularly interspaced short palindromic repeats) technology has revolutionized medicine as we know it and provides for another avenue of treatment for COVID-19. This tool exploits the Watson-Crick complementary base-pairing strategy, followed by the subsequent utilization of Cas9 (a bacterial-derived nuclease) to cleave or fix specific point mutations present on a target gene [60]. With the safe and efficient delivery of CRISPR-Cas9 being a major challenge, nanomedicine can provide a possible solution. CuNPs, due to their many advantageous properties [61], pose as attractive means for delivery of therapeutic genes, including CRISPR-Cas9 mediated gene therapy to target various diseased sites due to SARS-CoV-2. CRISPR-Cas9 has previously portrayed perfect binding specificity to targeted sites, leading to its beneficial role in this therapeutic approach [62]. 
The COVID-19 pandemic has required unusual or "out of the ordinary" research to be conducted and to think "out of the box". This was seen in a study performed by a team at Stanford, utilizing Cas13, instead of Cas9, as the virus-killing enzyme. This strategy is known as prophylactic anti-viral CRISPR in human cells or PAC-MAN [63]. This study portrayed PAC-MAN's ability to target $90 \%$ of all coronaviruses, with only six CRISPR RNA (crRNA) moieties. An effective means of delivery is perceived to lay with NPs. Once again, CuNPs possess the great ability to be conjugated with various pharmacologically active agents, the CRISPR technology included. Such novel approaches could potentially hold the key to treating COVID-19 and mitigating the high infection and mortality rates noted daily.

\section{Conclusion}

The efficacy of a specific study, or the level of protection, which is conferred to a vast population, is a significant factor in therapeutics. However, it is not the entire story. The efficacy of one study may be the stepping-stone to more significant achievements in another. The present review paper highlights $\mathrm{Cu}$ and CuNPs as potential therapeutics and therapeutic nanocarriers against COVID-19. The variations by which these are used are not limited, and thus the versatility of these NPs has become attractive in the development of treatment strategies, vaccines, and PPE. Nanosponges have also been identified to have a potential role in reducing this disease burden. Current challenges have hence necessitated novel studies and trials to be conducted, and exploitation of the propositions put forward above.

\section{Funding}

This research was funded by the National Research Foundation, South Africa, for funding (Grant number 120455).

\section{Acknowledgments}

This research has no acknowledgment.

\section{Conflicts of Interest}

The authors declare no conflict of interest.

\section{References}

1. Cascella, M.; Rajnik, M.; Cuomo, A.; Dulebohn, S.; Di Napoli, R. Features, evaluation and Treatment of Coronavirus (COVID-19). In: StatPearls [Internet]. Treasure Island (FL): StatPearls Publishing 2020.

2. Mehta, P.; McAuley, D.; Brown, M.; Sanchez, E.; Tattersall, R.; Manson, J. COVID-19: consider cytokine storm syndromes and immunosuppression. Lancet 2020, 395, 1033-1034, https://doi.org/10.1016/S01406736(20)30628-0.

3. Vincent, M.; Duval, R.; Hartemann, P.; Engels-Deutsch, M. Contact killing and antimicrobial properties of copper. J. Appl. Microbiol. 2018, 124, 1032-1046, https://doi.org/10.1111/jam.13681.

4. Doremalen, N.; Bushmaker, T.; Morris, D.; Holbrook, M.; Gamble, S.; Williamson, B.; Tamin, A.; Harcourt, J.; Thornburg, N.T.; Gerber, S.I.; Lloyd-Smith, J.O.; de Wit, E.; Munster, V.J. Aerosol and surface stability of CoV-19 (SARS-CoV-2) compared to SARS-CoV-1. N. Engl.J. Med. 2020, 382, 1564-1567, https://doi.org/10.1056/NEJMc2004973.

5. Raha, S.; Mallick, R.; Basak, S.; Duttaroy, A. Is copper beneficial for COVID-19 patients? Med. Hypotheses 2020, 142, https://doi.org/10.1016/j.mehy.2020.109814.

6. Maiyo, F.; Singh. M. Polymerized Selenium nanoparticles for Folate-Receptor Targeted Delivery of antiLuc-siRNA: Potential for Gene Silencing. Biomedicines 2020, 8 , https://doi.org/10.3390/biomedicines8040076. 
7. Zhang, Q.; Honko, A.; Zhou, J.; Gong, H.; Downs, S.; Vasquez, J.; Fang, R.; Gao, W.; Griffiths, A.; Zhang, L. Cellular Nanosponges Inhibit SARS-CoV-2 Infectivity. Nano Lett. 2020, 20, 5570-5574, https://doi.org/10.1021/acs.nanolett.0c02278.

8. Tinkle, S.; McNeil, S.; Mühlebach, S.; Bawa, R.; Borchard, G.; Barenholz, Y.; Tamarkin, L.; Desai, N. Nanomedicines: addressing the scientific and regulatory gap. Ann. $N$ Y. Acad. Sci. 2014, 1313, 35-56.

9. Ahmed, S.; Quadeer, A.; McKay, M. Preliminary Identification of Potential Vaccine Targets for the COVID19 Coronavirus (SARS-CoV-2) Based on SARS-CoV Immunological Studies. Viruses 2020, 12, https://doi.org/10.3390/v12030254.

10. Kannan, S.; Ali, P.; Sheeza, A.; Hemalatha, K. COVID-19 (Novel Coronavirus 2019) - Recent trends. Eur. Rev. Med. Pharmacol. Sci. 2020, 24, 2006-2011, https://doi.org/10.26355/eurrev_202002_20378.

11. Gui, M.; Song, W.; Zhou, H.; Xu, J.; Chen, S.; Xiang, Y.; Wang, X. Cryo-electron microscopy structures of the SARS-CoV spike glycoprotein reveal a prerequisite conformational state for receptor binding. Cell Res. 2016, 27, 119-129, https://doi.org/10.1038/cr.2016.152.

12. Song, W.; Gui, M.; Wang, X.; Xiang, Y. Cryo-EM structure of the SARS coronavirus spike glycoprotein in complex with its host cell receptor ACE2. PLoS Pathog. 2018, 14, https://doi.org/10.1371/journal.ppat.1007236.

13. Zhou, P.; Yang, X.; Wang, X.; Hu, B.; Zhang, L.; Zhang, W.; Si, H.; Zhu, Y.; Li, B.; Huang,H-D.; Chen, HD.; Chen, J.; Luo, Y.; Guo, H.; Jiang, R.-D.; Liu, M.-Q.; Chen, Y.; Shen, X.-R.; Wang, X.; Zheng, X.-S.; Zhao, K.; Chen, Q.-J.; Deng, F.; Liu, L.-L.; Yan, B.; Zhan, F.-X.; Wang, Y.-Y.; Xiao, G.-F.; Shi, Z.-L. A pneumonia outbreak associated with a new coronavirus of probable bat origin. Nature 2020, 579, 270273, https://doi.org/10.1038/s41586-020-2012-7.

14. Lan, J; Ge, J; Yu, J; Shan, S; Zhou, H; Fan, S; Zhang, Q; Shi, X.; Wang, Q.; Wang, X. Structure of the SARS-CoV-2 spike receptor-binding domain bound to the ACE2 receptor. Nature 2020, 581, 215-220, https://doi.org/10.1038/s41586-020-2180-5.

15. Wu, J.; Leung, K.; Leung, G. Nowcasting and forecasting the potential domestic and international spread of the 2019-nCoV outbreak originating in Wuhan, China: a modelling study. Lancet 2020, 395, 689-697, https://doi.org/10.1016/S0140-6736(20)30260-9.

16. Tisoncik, J.; Korth, M.; Simmons, C.; Farrar, J.; Martin, T.; Katze, M. Into the Eye of the Cytokine Storm. Microbiol. Mol. Biol. Rev. 2020, 76, 16-32, https://doi.org/10.1128/MMBR.05015-11.

17. Huang, C.; Wang, Y.; Li, X; Ren, L.; Zhao, J.; Hu, Y.; Zhang, L.; Fan, G.;Xu, J.; Gu, X.; Cheng, Z.; Yu, T.; Xia, J.; Wei, Y.; Wu, W.; Xie, X.; Yin, W,; Li, H,; Liu, M.; Xiao, Y.; Gao, H,; Guo, L.; Xie, J.; Wang, G.; Jiang, R.; Gao, Z.; Jin, Q.; Wang, J.; Cao, B. Clinical features of patients infected with 2019 novel coronavirus in Wuhan, China. Lancet 2012, 395, 497-506, https://doi.org/10.1016/S0140-6736(20)301835.

18. Graham, R.; Becker, M.; Eckerle, L.; Bolles, M.; Denison, M.; Baric, R. A live, impaired-fidelity coronavirus vaccine protects in an aged, immunocompromised mouse model of lethal disease. Nature Medicine 2012, 18, 1820-1826, https://doi.org/10.1038/nm.2972.

19. Wu, L.; Wang, N.; Chang, Y.; Tian, X.; Na, D.; Zhang, L.; Zheng, L.; Lan, T.; Wang, L.; Liang, G. Duration of Antibody Responses after Severe Acute Respiratory Syndrome. Emerging Infectious Diseases 2007, 13, 1562-1564, https://doi.org/10.3201/eid1310.070576.

20. Warnes, S.; Little, Z.; Keevil, C. Human Coronavirus 229E Remains Infectious on Common Touch Surface Materials. mBio 2015, 6, e01697-15, https://doi.org/10.1128/mBio.01697-15.

21. Horie, M.; Ogawa, H.; Yoshida, Y.; Yamada, K.; Hara, A.; Ozawa, K.; Matsuda, S.; Mizota, C.; Yamamoto, Y.; Yamada, M.; Nakamura, K.; Imai, K. Inactivation and morphological changes of avian influenza virus by copper ions. Arch.Virol. 2008, 153, 1467-1472, https://doi.org/10.1007/s00705-008-0154-2.

22. Fujimori, Y.; Sato, T.; Hayata, T.; Nagao, T.; Nakayama, M.; Nakayama, T.; Sugamata, R.; Suzuki, K. Novel Anti-viral Characteristics of Nanosized Copper(I) Iodide Particles Showing Inactivation Activity against 2009 Pandemic H1N1 Influenza Virus. Appl. Environ. Microbiol. 2011, 78, 951-955, https://doi.org/10.1128/AEM.06284-11.

23. Rupp, J.; Locatelli, M.; Grieser, A.; Ramos, A.; Campbell, P.; Yi, H.; Steel, J.; Burkhead, J.; Bortz, E. Host Cell Copper Transporters CTR1 and ATP7A are important for Influenza A virus replication. Virol. J. 2017, 14, https://doi.org/10.1186/s12985-016-0671-7.

24. Nève, J.; Fontaine, J.; Peretz, A.; Famaey, J. Changes in zinc, copper and selenium status during adjuvantinduced arthritis in rats. Agents Actions 1988, 25, 146-155, https://doi.org/10.1007/BF01969106.

25. Masters, P.S. The molecular biology of coronaviruses. Adv. Virus Res. 2006, 66, 193-292, https://doi.org/10.1016/S0065-3527(06)66005-3.

26. Cherukuri, S.; Potla, R.; Sarkar, J.; Nurko, S.; Harris, Z.; Fox, P. Unexpected role of ceruloplasmin in intestinal iron absorption. Cell Metab. 2005, 2, 309-319, https://doi.org/10.1016/j.cmet.2005.10.003.

27. Andreou, A.; Trantza, S.; Filippou, D.; Sipsas, N.; Tsiodras, S. COVID-19: The Potential Role of Copper and N-acetylcysteine (NAC) in a combination of Candidate Antiviral Treatments against SARS-CoV-2. In Vivo 2020, 34, 1567-1588, https://doi.org/10.21873/invivo.11946.

28. Lönnerdal, B. Bioavailability of copper. Am. J. Clin. Nutr. 1996, 63, 821S-829S, https://doi.org/10.1093/ajcn/63.5.821. 
29. Ong, S.; Tan, Y.; Chia, P.; Lee, T.; Ng, O.; Wong, M.; Marimuthu, K. Air, Surface Environmental, and Personal Protective Equipment Contamination by Severe Acute Respiratory Syndrome Coronavirus 2 (SARS-CoV-2) From a Symptomatic Patient. JAMA 2020, 323, 1610-1612, https://doi.org/10.1001/jama.2020.3227.

30. Mohanraj, V.; Chen, Y. Nanoparticles-A review. Trop. J. Pharm. Res. 2007, 5, 561-673, https://doi.org/10.4314/tjpr.v5i1.14634.

31. Baetke, S.; Lammers, T.; Kiessling, F. Applications of nanoparticles for diagnosis and therapy of cancer. $B r$. J. Radiol. 2015, 88, https://doi.org/10.1259/bjr.20150207.

32. Weiss, C.; Carriere, M.; Fusco, L.; Capua, I.; Regla-Nava, J.; Pasquali, M.; Scott, J.; Vitale, F.; Unal, M.A.; Mattevi, C.; Bedognetti, D.; Merkoci, A.; Tasciotti, E.; Yilmazer, A.; Gogotsi, Y.; Stellaci, F.; Delogu, L.G. Toward Nanotechnology-Enabled Approaches against the COVID-19 Pandemic. ACS Nano 2020, 14, 63836406, https://doi.org/10.1021/acsnano.0c03697.

33. Maney, V.; Singh, M. The synergism of Platinum-Gold bimetallic nanoconjugates enhance 5-Fluorouracil delivery in vitro. Pharmaceutics 2019, 11, https://doi.org/10.3390/pharmaceutics11090439.

34. Joob, B.; Wiwanitkit, V. Nanodiagnosis for Diagnosing COVID-19: a Brief Review. Lett. Appl. NanoBiosci. 2020, 9, 1578-1582, https://doi.org/10.33263/LIANBS94.15781582.

35. Narasaiah, B.P.; Mandal, B.K. Bio-fabricated $\mathrm{CuO}$ NPs as green catalyst towards remediation of environmental pollutants. Lett. Appl. NanoBiosci. 2019, 8, 597-603. https://doi.org/10.33263/LIANBS83.597603.

36. Ullah, N.; Ullah, A.; Rasheed, S. Green synthesis of copper nanoparticles using extract of Dicliptera Roxburghiana, their characterization and photocatalytic activity against methylene blue degradation. Lett. Appl. NanoBiosci. 2020, 9, 897-901, https://doi.org/10.33263/LIANBS91.897901.

37. Ingle, A.P.; Duran, N.; Rai, M. Bioactivity, mechanism of action, and cytotoxicity of copper-based nanoparticles: a review. Appl. Microbiol. Biotechnol. 2014, 98, 1001-1009, https://doi.org/10.1007/s00253013-5422-8.

38. Wang, L. Synthetic methods of CuS nanoparticles and their applications for imaging and cancer therapy. RSC Adv. 2016, 6, 82596-82615, https://doi.org/10.1039/C6RA18355G.

39. Sierra-Ávila, R.; Pérez-Alvarez, M.; Cadenas-Pliego, G.; Comparán-Padilla, V.; Ávila-Orta, C.; PérezCamacho, O.; Jiménez-Regalado, E.; Hernández-Hernández, E.; Jiménez-Barrera, R. Synthesis of Copper Nanoparticles using Mixture of Allylamine and Polyallylamine. J. Nanomater. 2015, 2015, https://doi.org/10.1155/2015/367341.

40. Tadjarodi, A.; Roshani, R. A green synthesis of copper oxide nanoparticles by mechanochemical method. Curr. Chem. Lett. 2014, 3, 215-220, https://doi.org/10.5267/j.ccl.2014.7.001.

41. Sankar, R.; Maheswari, R.; Karthik, S.; Shivashangari, K.; Ravikumar, V. Anticancer activity of Ficus religiosa engineered copper oxide nanoparticles. Mater. Sci.Eng.C. 2014, 44, 234-239, https://doi.org/10.1016/j.msec.2014.08.030.

42. Sportelli, M.; Izzi, M.; Kukushkina, E.; Hossain, S.; Picca, R.; Ditaranto, N.; Cioffi, N. Can Nanotechnology and Materials Science Help the Fight against SARS-CoV-2? Nanomater. 2020, 10, https://doi.org/10.3390/nano10040802.

43. Hang, X.; Peng, H.; Song, H.; Qi, Z.; Miao, X.; Xu, W. Anti-viral activity of cuprous oxide nanoparticles against Hepatitis C Virus in vitro. J. Virol. Meth. 2015, 222, 150-157, https://doi.org/10.1016/j.jviromet.2015.06.010.

44. Tavakoli, A.; Hashemzadeh, M. Inhibition of herpes simplex virus type 1 by copper oxide nanoparticles. J. Virol. Meth. 2020, 275, https://doi.org/10.1016/j.jviromet.2019.113688.

45. Swaminathan, S.; Cavalli, R.; Trotta, F. Cyclodextrin-based nanosponges: a versatile platform for cancer nanotherapeutics development. Wiley Interdiscip. Rev. Nanomed. Nanobiotechnol. 2016, 8, 579-601.

46. Tannous, M.; Trotta, F.; Cavalli, R. Nanosponges for combination drug therapy: state-of-the-art and future directions. Nanomedicine 2020, 15, 643-646, https://doi.org/10.2217/nnm-2020-0007

47. Rosado, C.; Kondos, S.; Bull, T.; Kuiper, M.; Law, R.; Buckle, A.; Voskoboinik, I.; Bird, P.I.; Trapani, J.A.; Whisstock, J.C.; Dunstone, M.A.; Bird, P. The MACPF/CDC family of pore-forming toxins. Cell. Microbiol. 2008, 10, 1765-1774.

48. Hu, C.; Fang, R.; Copp, J.; Luk, B.; Zhang, L. A biomimetic nanosponge that absorbs pore-forming toxins. Nat. Nanotechnol. 2013, 8, 336-340, https://doi.org/10.1038/nnano.2013.54.

49. Patra, J.; Das, G.; Fraceto, L.; Campos, E.; Rodriguez-Torres, M.; Acosta-Torres, L.; Diaz-Torres, L.; Grillo, R.; Swamy, M.R.; Sharma, S.; Habtemariam, S.; Shin, H.-S. Nano based drug delivery systems: recent developments and future prospects J. Nanobiotechnol. 2018, 16, https://doi.org/10.1186/s12951-018-03928 .

50. Mirza, A.; Siddiqui, F. Nanomedicine and drug delivery: a mini-review. Int. Nano Lett. 2014, 4, https://doi.org/10.1007/s40089-014-0094-7.

51. Lu, H.; Wang, J.; Wang, T.; Zhong, J.; Bao, Y.; Hao, H. Recent Progress on Nanostructures for Drug Delivery Applications. J. Nanomater. 2016, 2016, https://doi.org/10.1155/2016/5762431. 
52. Dormont, F.; Brusini, R.; Cailleau, C.; Reynaud, F.; Peramo, A.; Gendron, A.; Mougin, J.; Gaudin, F.; Varna, M.; Couvreur, P. Squalene-based multidrug nanoparticles for improved mitigation of uncontrolled inflammation in rodents. Sci. Adv. 2020, 6, https://doi.org/10.1126/sciadv.aaz5466.

53. Lai, C.; Shih, T.; Ko, W.; Tang, H.; Hsueh, P. Severe acute respiratory syndrome coronavirus 2 (SARSCoV-2) and coronavirus disease-2019 (COVID-19): The epidemic and the challenges. Int. J. Antimicrob. Agents 2020, 55, https://doi.org/10.1016/j.ijantimicag.2020.105924.

54. Wang, M.; Cao, R.; Zhang, L.; Yang, X.; Liu, J.; Xu, M.; Shi, Z.; Hu, Z.; Zhong, W.; Xiao, G. Remdesivir and chloroquine effectively inhibit the recently emerged novel coronavirus (2019-nCoV) in vitro. Cell Res. 2020, 30, 269-271, https://doi.org/10.1038/s41422-020-0282-0.

55. Wang, Z.; Chen, X.; Lu, Y.; Chen, F.; Zhang, W. Clinical characteristics and therapeutic procedure for four cases with 2019 novel coronavirus pneumonia receiving combined Chinese and Western medicine treatment. BioSci. Trends 2020, 14, 64-68, https://doi.org/10.5582/bst.2020.01030.

56. Vandenberghe, L. COVID-19: Gene Transfer to the Rescue? Hum. Gene Ther. 2020, 31, 605-607, https://doi.org/10.1089/hum.2020.29125.lhv.

57. Amer, M. Gene therapy for cancer: present status and future perspective. Mol.Cell. Ther. $2014,2$.

58. Koenig, O.; Walker, T.; Perle, N.; Zech, A.; Neumann, B.; Schlensak, C.; Wendel, H.; Nolte, A. New Aspects of Gene-Silencing for the Treatment of Cardiovascular Diseases. Pharmaceuticals 2013, 6, 881-914, https://doi.org/10.3390/ph6070881.

59. Wirth, T.; Parker, N.; Ylä-Herttuala, S. History of gene therapy. Gene 2013, 525, 162-169, http://dx.doi.org/10.1016/j.gene.2013.03.137.

60. Wei, T.; Cheng, Q.; Min, Y.; Olson, E.; Siegwart, D. Systemic nanoparticle delivery of CRISPR-Cas9 ribonucleoproteins for effective tissue specific genome editing. Nat. Commun. 2020, 11, https://doi.org/10.1038/s41467-020-17029-3.

61. Cheung, A.; Chow, C.; Zhang, J.; Zhou, Y.; Huang, T.; Ng, K.; Or, T.; Yao, Y. Specific targeting of point mutations in EGFR L858R-positive lung cancer by CRISPR/Cas9. Lab. Investig. 2018, 98, 968-976, https://doi.org/10.1038/s41374-018-0056-1.

62. Tao, Y.; Hou, X.; Zuo, F.; Li, X.; Pang, Y.; Jiang, G. Application of nanoparticle-based siRNA and CRISPR/Cas9 delivery systems in gene-targeted therapy. Nanomedicine 2019, 14, 511-514, https://doi.org/10.2217/nnm-2018-0522.

63. Abbott, T.; Dhamdhere, G.; Liu, Y.; Lin, X.; Goudy, L.; Zeng, L.; Chemparathy, A.; Chmura, S.; Heaton, N.; Debs, R.; Pande, T.; Endy, D.; La Russa, M.; Lewis, D.; Qi, L. Development of CRISPR as an Antiviral Strategy to Combat SARS-CoV-2 and Influenza. Cell 2020, 181, 865-876, https://doi.org/10.1016/j.cell.2020.04.020. 